Annuaire suisse de politique de développement

23-1 | 2004

Faits et statistiques 2004

\title{
11. Politique de paix et politique de sécurité
}

\section{Christoph Stamm}

\section{OpenEdition}

\section{Journals}

Édition électronique

URL : http://journals.openedition.org/aspd/481

DOI : $10.4000 /$ aspd.481

ISSN : 1663-9669

\section{Éditeur}

Institut de hautes études internationales et du développement

\section{Édition imprimée}

Date de publication : 1 avril 2004

Pagination : 195-208

ISSN : 1660-5934

\section{Référence électronique}

Christoph Stamm, «11. Politique de paix et politique de sécurité », Annuaire suisse de politique de développement [En ligne], 23-1 | 2004, mis en ligne le 12 mars 2010, consulté le 08 septembre 2020 URL : http://journals.openedition.org/aspd/481 ; DOI : https://doi.org/10.4000/aspd.481 


\section{Politique de paix et politique de sécurité*}

E

N 2003, le Parlement suisse a été appelé à se prononcer sur deux créditscadres et sur une loi fédérale en rapport avec la promotion civile de la paix. Si nul n'a contesté le contenu des projets, il en fut autrement pour le montant des crédits: le Conseil des Etats n'a pas suivi la proposition du Conseil fédéral et s'est prononcé pour une réduction des dépenses.

Les efforts visant à consolider la paix au Sri Lanka furent en 2003 l'une des priorités des activités suisses au service de la paix. Divers organismes suisses sont en effet intervenus à différents niveaux pour faire progresser un processus entamé en 2001 déjà.

Outre la Division politique IV du Département fédéral des affaires étrangères (DFAE) et le Département fédéral de la défense, de la population et des sports (DDPS), la Direction du développement et de la coopération $(D D C)^{l}$ et des organisations non gouvernementales ${ }^{2}$ cuvrent pour la résolution de conflits. Preuve en sont le projet FAST d'alerte rapide et la promotion de la paix dans la province d'Aceh, en Indonésie.

Les acteurs suisses de la politique étrangère ont également repris à leur compte la notion de "sécurité humaine», qui a fait son apparition dans les années 1990 et qui place l'individu en tête des priorités en matière de sécurité. La sécurité humaine préside ainsi à des activités que la Division politique IV du DFAE mène dans différents domaines. Ces activités incluent notamment le Réseau de la sécurité humaine, l'élimination des mines antipersonnel partout dans le monde et le contrôle du commerce international d'armes de petit calibre.

Plus d'une décennie après la dissolution de l'Union soviétique, les sites contaminés par son industrie de l'armement n'ont toujours pas été assainis, bien qu'ils représentent un grave danger pour la sécurité, la santé et l'environnement. La Suisse a accordé un crédit pour faciliter l'application de la Convention sur les armes chimiques et pour contribuer au démantèlement de l'arsenal russe de telles armes.

En mai 2003, la peuple et les Etats ont approuvé la réforme intitulée Armée XXI, qui doit adapter l'armée suisse à la nouvelle donne dans le domaine de la sécurité. A l'avenir, la sécurité de la Suisse devrait donc être davantage assurée par une coopération internationale approfondie.

* Par Christophe Stamm, politologue.

1 En 2003, la DDC a présenté des directives pour le développement de la paix. Dans sa Stratégie 2010, elle avait d'ailleurs déjà rangé la prévention et la résolution des crises parmi les cinq thèmes prioritaires de la coopération. Voir Annuaire 2004, n 1, chap. 2, «Coopération avec les pays en développement».

2 Celles-ci bénéficient parfois d'un soutien politique et financier substantiel de la part de la Confédération. Pour un aperçu de ce soutien aux ONG, voir Claude André Ribaux, Friedensförderung und Konfliktbearbeitung in der Schweiz. Eine Bestandesaufnahme, Saint-Gall, Typotron, 2003. 


\subsection{Projet de loi et crédits-cadres ayant trait à la politique de paix}

$\square$ Loi fédérale et crédit-cadre concernant des mesures

de gestion civile des conflits et de promotion des droits de l'homme

Le projet de loi que le Conseil fédéral a présenté en 2002 sur des mesures de promotion civile de la paix et de renforcement des droits de l'homme n'a guère été contesté par les Chambres ${ }^{3}$. Le Conseil national a adopté la nouvelle loi en mars 2003 par 107 voix contre 24. En septembre, le Conseil des Etats l'approuva à l'unanimité après lui avoir apporté quelques légères modifications ${ }^{4}$.

Le Conseil national a aussi accepté à une nette majorité, 120 voix contre 27 , d'ouvrir pour quatre années au moins un crédit-cadre de 240 millions de francs allant de pair avec la loi. L'adoption de ce crédit a suscité un débat plus nourri au Conseil des Etats. La conseillère fédérale Micheline Calmy-Rey a eu beau défendre le montant prévu de 240 millions de francs en arguant que le Conseil fédéral avait fait de la promotion civile de la paix une priorité stratégique et que les moyens supplémentaires consacrées à cet effort ont été épargnés dans d'autres secteurs de son département (DFAE), rien n'y fit. Le Conseil des Etats s'est opposé, par 27 voix contre 10, au Conseil fédéral et a décidé, par 24 voix contre 3, d'abaisser le crédit à 200 millions de francs. En décembre 2003, les deux Conseils ont fini par trouver un consensus : le crédit-cadre adopté se monte à 220 millions de francs.

$\mathbb{D}$ Annuaire 2003, $\mathrm{n}^{\circ}$ 1, Loi fédérale sur des mesures de promotion civile de la paix et de renforcement des droits de l'homme (pp. 7-8).

\section{Crédit-cadre pour des mesures}

relatives à la promotion civile de la paix au DDPS

En décembre 2002, le Conseil fédéral a présenté au parlement un message concernant l'ouverture d'un crédit-cadre pour des mesures relatives à la promotion civile de la paix au DDPS (Département fédéral de la défense, de la population et des sports $)^{5}$. Ce crédit de plus de 180 millions de francs ouvert pour la période allant de 2004 à 2007 servira à financer les activités civiles de promotion de la paix du DDPS. Ces activités englobent principalement le Centre de politique de sécurité, le Centre international de déminage humanitaire et le Centre pour le contrôle démocratique des forces armées, à Genève, et l'International Relations and Security Network, de l'EPF de Zurich (qui se verront allouer respectivement $15 \%, 18 \%, 23 \%$ et $19 \%$ du budget total). Le crédit permettra aussi de financer divers projets dans le cadre du Partenariat pour la paix

3 Conseil fédéral, Message concernant l'ouverture d'un crédit-cadre pour des mesures de gestion civile des conflits et de promotion des droits de l'homme du 23 octobre 2002 (FF 2002 7395) et Message concernant la loi fédérale sur des mesures de promotion civile de la paix et de renforcement des droits de l'homme du 23 octobre 2002 (FF 2002 7063).

4 Le Conseil des Etats a biffé la création d'une commission d'experts prévue dans la loi et offert la possibilité à la Confédération de soutenir des institutions scientifiques. Un postulat a par ailleurs demandé au Conseil fédéral de donner une structure interdépartementale à la conduite politique et à la coordination de la promotion civile de la paix et de la gestion des conflits au niveau de la Confédération. Le Conseil fédéral doit également adresser un rapport au Parlement sur ces activités (Postulat 03.3178).

5 Conseil fédéral, Message concernant l'ouverture d'un crédit-cadre pour des mesures relatives à la promotion civile de la paix au DDPS du 9 décembre 2002 (FF 2003 561). 
de l'OTAN ainsi que des programmes bilatéraux ou multilatéraux de coopération ou de soutien. Pendant les quatre années à venir, les services compétents vont s'attacher à poursuivre et à consolider les activités actuelles.

DD Annuaire 2003, $\mathrm{n}^{\circ}$ 1, point 11.3., «Institutions suisses de promotion de la paix » (pp. 201-202).

La Confédération ambitionne de construire à Genève, d'ici 2007, une Maison de la paix. Elle souhaite y regrouper les différents centres genevois et mettre des locaux à la disposition des différentes institutions qui œuvrent dans un domaine connexe. Selon le Conseil fédéral, ce projet permettra d'étendre l'aura des institutions concernées, d'accroître leur efficacité, d'intensifier leurs contacts avec d'autres institutions présentes à Genève et de renforcer le rôle de Genève dans la politique de sécurité et de paix. Les ressources nécessaires à la construction de la Maison de la paix n'étaient pas comprises dans le crédit-cadre soumis au parlement car elles feront l'objet d'un futur message sur l'immobilier.

Aucune des deux Chambres ne s'est fait prier pour adopter le crédit-cadre: le Conseil national l'a approuvé en mars 2003 par 122 voix contre 10 et le Conseil des Etats l'a adopté à l'unanimité en septembre 2003 après lui avoir apporté quelques retouches.

\subsection{Principales activités relevant de la politique de paix}

\section{$\square$ Processus de paix laborieux au Sri Lanka}

Grâce aux bons offices de la Norvège et sous la pression des Etats-Unis et de l'Inde, le cessez-le-feu conclu en février 2002 entre le gouvernement et les Tigres de libération tamouls (LTTE) a conduit à une désescalade dans la guerre civile qui agite le Sri Lanka depuis 1983. Ce conflit a jusqu'ici fait 70'000 victimes humaines et 1,5 million de réfugiés.

Après plusieurs rondes de négociations entre le gouvernement et les LTTE, le processus de paix était arrivé dans une impasse au printemps 2003, avant une percée politique décisive. Depuis, la situation des droits de l'homme ${ }^{6}$ s'est quelque peu améliorée, mais l'insécurité qui règne encore freine la reconstruction. Autrement dit, les bienfaits de la paix se font attendre ${ }^{7}$.

La Suisse ayant accueilli plus de 35'000 réfugiés tamouls ces vingt dernières années, la politique étrangère réserve une place particulière au Sri Lanka. Voici quelques exemples qui illustrent l'engagement de diverses institutions suisses dans le processus de paix:

๑ Le Département fédéral des affaires étrangères (DFAE) finance, avec l'Allemagne, les activités du Resource Network for Conflict Studies and Transformation Sri Lanka (RNCST), mis en place par le Berghof-Zentrum für Konfliktforschung de Berlin. Le travail du RNCST vise à développer les capacités nécessaires pour assurer une gestion constructive du conflit sri-lankais à tous les niveaux (tracks ${ }^{8}$ dans le projet) ainsi que de créer des forums, des cadres de dialogue et de compréhension mutuelle. Diverses mesures sont

6 Amnesty International, Rapport annuel 2003, Francfort, Fischer, 2003.

7 «Die im Juni 2003 von der internationalen Gebergemeinschaft gesprochenen 4,5 Milliarden US-Dollar können noch nicht abgerufen werden », Wochenzeitung, 11 septembre 2003.

8 Les «tracks I à III» désignent différentes approches complémentaires dans la gestion du conflit. 
destinées à compléter et à renforcer les potentiels déjà existants sur place en matière de formation, de recherche et de dialogue ${ }^{9}$.

๖ Fin 2002, les parties ont convenu de rechercher une solution au conflit ethnopolitique en explorant les voies du fédéralisme, pour mettre fin à la forte centralisation dont souffre le fonctionnement de l'Etat. La Suisse appuie les efforts des partenaires sri-lankais en portant à leur connaissance ses expériences dans des domaines tels que le droit constitutionnel, le fédéralisme, les structures décentralisées et l'administration civile. Le DFAE et l'Institut du fédéralisme de l'Université de Fribourg proposent leurs conseils et organisent des cours sur ces sujets. En 2003, plusieurs délégations réunissant des ministres sri-lankais, des parlementaires, des représentants des LTTE et des journalistes sont venues en Suisse pour apprendre à mieux connaître le pays et son système fédéral ${ }^{10}$.

- Avec l'appui financier du DFAE, la Fondation suisse de déminage (FSD) a établi une carte exhaustive des zones minées à l'attention du Programme des Nations unies pour le développement (PNUD), formé des auxiliaires nationaux et mis à disposition des équipes d'urgence pour des opérations de déminage ${ }^{11}$.

๖ Un projet dirigé par Helvetas s'est fixé pour objectif de promouvoir la réconciliation, la paix et le développement dans le nord-est du Sri Lanka ${ }^{12}$. Pour favoriser la paix, il entend surtout atténuer les conflits et encourager la compréhension mutuelle en misant sur la collaboration entre les groupes de population et sur des rencontres culturelles et sportives. Des réseaux locaux réunissant des ONG œuvrant pour la paix devraient par ailleurs militer pour une résolution pacifique des conflits auprès des dirigeants politiques et autres responsables.

$\square$ Organisant périodiquement des tables rondes, le Centre pour la promotion de la paix (KOFF) offre la possibilité aux représentants des services de l'Etat et d'organisations non gouvernementales d'échanger régulièrement des informations sur les tendances actuelles et sur les enjeux du processus de paix au Sri Lanka ${ }^{13}$.

\section{$\square$ Echec des efforts de paix dans la province d'Aceh, en Indonésie}

Le Mouvement Aceh libre (GAM) mène depuis 1976 une lutte armée pour obtenir l'indépendance de la province indonésienne d'Aceh. Située à l'extrémité nord du pays, cette province est riche en matières premières dont l'exploitation ne bénéficie toutefois guère à la population locale. L'espoir des séparatistes a été ranimé par le détachement du Timor-Oriental et la création, en 2002, de l'Etat de Timor-Leste. De leur côté, le gouvernement indonésien et l'armée se déclarent prêts à recourir à tous les moyens pour éviter une sécession de la province.

Deux ONG qui œuvrent pour la promotion de la paix et le respect des droits de l'homme et dont le siège est en Suisse travaillent à la résolution de ce grave pro-

9 Berghof Forschungszentrum für konstruktive Konfliktbearbeitung: <www.berghof-center.org >. Berghof Foundation for Conflict Studies, <www.berghof-foundation.lk>.

10 Le DFAE souhaite mieux exploiter l'avantage comparatif que représente l'expérience positive du fédéralisme suisse pour promouvoir la paix.

11 Fondation suisse de déminage, Rapport annuel 2002, <www.mineaction.ch>.

12 Helvetas, <www.helvetas.ch>.

13 KOFF Newsletter, $\mathrm{n}^{\circ}$ 7, 2003. 
blème. Il s'agit du Centre for Humanitarian Dialogue (HDC) ${ }^{14}$, une institution genevoise, et des Brigades de paix internationales $(\mathrm{PBI})^{15}$, une organisation internationale dont le bureau suisse se trouve à Fribourg. Ces deux organismes ont des approches de la politique de paix différentes mais néanmoins complémentaires.

Le travail du Centre for Humanitarian Dialogue vise surtout à promouvoir les principes humanitaires, à prévenir les conflits et à atténuer leurs conséquences. En 2003, la Confédération a alloué 1 million de francs à cet organisme pour soutenir ses activités.

En 2000, le centre a commencé à promouvoir le dialogue entre le gouvernement indonésien et le GAM. Dès 2001, les parties en conflit se sont rencontrées pour s'entretenir sous l'égide de l'institution genevoise et ont fini par se déclarer prêtes à définir des mécanismes de résolution du conflit. A fin 2002, après la mise en ouvre de diverses mesures destinées à établir la confiance et d'autres rondes de négociations, les rebelles et le gouvernement indonésien ont signé à Genève un accord de cessez-le-feu qui accorde l'autonomie à la province d'Aceh. En janvier 2003, des groupes tripartites, réunissant des observateurs du HDC et des représentants du gouvernement indonésien et du GAM, sont entrés en action pour vérifier le respect du cessez-le-feu. Plusieurs zones de paix ont pu être délimitées et des personnes déplacées ont pu regagner leurs foyers. Le HDC n'a cependant pas réussi à éviter que le processus de paix s'enlise au printemps 2003. Le Mouvement Aceh libre et l'armée indonésienne ont tous deux violé le plan de paix établi et rejeté tout compromis. L'entrée en vigueur de la loi martiale en mai 2003 et de nouveaux combats ont anéanti tout espoir d'une paix prochaine et obligé le Centre for Humanitarian Dialogue à se retirer de la région. Le principal projet de ce centre a ainsi échoué.

Les Brigades de paix internationales (PBI) interviennent dans les zones de crise et en guerre pour protéger les droits de l'homme et favoriser la gestion pacifique des conflits. Des équipes bénévoles se rendent sur place afin de créer des «espaces de paix» pour permettre aux organisations de la société civile qui axent leur travail sur la non-violence de continuer à promouvoir les droits de l'homme, la justice sociale et la gestion pacifique des conflits. PBI Suisse compte parmi les sections nationales les plus grandes et reçoit des appuis financiers de la part d'institutions publiques suisses. Celles-ci ont par exemple assuré $38 \%$ du budget des années 2001 et 2002. Pour sa part, PBI Suisse a versé $45 \%$ de ses ressources à Peace Brigades International, dont le siège est à Londres.

Les interventions des Brigades de paix répondent toujours à un appel émanant de groupements locaux de promotion des droits de l'homme. Et c'est suite à une telle demande que les PBI ont lancé leurs activités en Indonésie et ouvert des bureaux à Jakarta, à Banda Aceh et à Lhokseumawe (ces deux dernières villes étant situées dans la province d'Aceh). En 2003, le projet indonésien occupait 20 bénévoles, qui accompagnaient diverses personnes menacées pour les protéger et permettre à leurs groupements de poursuivre leurs activités malgré le climat de peur et de violence. Le bureau de Jakarta entretient aussi des contacts avec des services de l'Etat, des représentations étrangères et des organisations

14 Centre for Humanitarian Dialogue, <www.hdcentre.org $>$.

15 Brigades de paix internationales, $<w w w$.peacebrigades.org $>$. 
nationales et internationales, et observe l'évolution politique sur place. Après la reprise du conflit armé en juin 2003, le gouvernement indonésien a obligé les PBI à fermer leurs bureaux dans la province d'Aceh et à quitter la région.

\section{$\square$ Le système FAST}

Le système d'alerte précoce en cas de conflit, Early Recognition of Tension and Fact-Finding (FAST), a été mis sur pied en 1998 sur mandat de la DDC ${ }^{16}$. Dirigé par la Fondation suisse pour la paix, Swisspeace, ce projet a pour objectif d'identifier et de signaler les évolutions politiques à risque et les hausses de tension avant qu'elles ne dégénèrent en conflit armé. Ce système d'alerte doit permettre aux responsables politiques d'élaborer des stratégies cohérentes pour éviter ou contrer des tendances destructrices et de trouver les moyens de prévenir un conflit. FAST tient compte des besoins géographiques de la politique étrangère de la Suisse et le nombre des pays qu'il observe ne cesse de croître. En 2003, le projet a ainsi analysé 24 pays ou régions dans les parties du monde suivantes: région des Grands Lacs en Afrique, Corne de l'Afrique, Afrique australe, Asie centrale, Caucase et Balkans. Deux caractéristiques distinguent ce projet d'autres systèmes d'alerte: premièrement, il combine des méthodes quantitatives et qualitatives. Les informations quantitatives proviennent de l'analyse d'événements et des informations fournies par des réseaux locaux, tandis que les analyses qualitatives sont fournies par des experts des différents pays. Deuxièmement, FAST intervient dans le processus de décision politique et comble ainsi l'écart qui sépare l'alerte précoce (early warning) et l'action précoce (early action).

Des travaux sont actuellement en cours pour élaborer des modèles prédictifs sur le cadre conflictuel et coopératif dans les pays cibles. Avec la conclusion de partenariats scientifiques et la création d'une base institutionnelle internationale, le projet devrait se développer pour devenir «FAST International». En 2003, d'intenses négociations ont été menées à ce propos avec des organismes de développement d'autres pays.

Une évaluation externe de la Fondation suisse pour la paix a conclu que FAST a fourni, dans son secteur d'activités, un travail de pionnier sur le plan international ${ }^{17}$. L'évaluation regrette cependant que FAST soit peu présent au sein de la communauté scientifique internationale qui se consacre à l'étude de la paix ${ }^{18}$. Cette «discrétion» s'expliquerait par le manque de temps des scientifiques collaborant au projet et les exigences en matière de confidentialité du mandat attribué par la DDC.

16 Système FAST, <www.swisspeace.org/fast>

17 Evaluation der Schweizerischen Friedensstiftung «Swisspeace», réalisée par le Centre d'études de la science et de la technologie (CEST) sur mandat de l'Office fédéral de la formation professionnelle et de la technologie, <www.cest.ch>.

18 «Hier besitzt die Schweiz ein wissenschaftliches Produkt von absoluter Weltspitzenklasse, und viel zu wenige wissen es.» (Harald Müller, in Evaluation der Schweizerischen Friedenstiftung «Swisspeace», op. cit.). 


\section{$\square$ Sécurité humaine - Une notion nouvelle}

Si la politique de sécurité se concentrait par le passé sur le cadre étatique ou, plus exactement, sur les relations entre Etats et sur la situation mondiale (sécurité nucléaire), depuis les années 1990, c’est la sécurité individuelle qui occupe une place importante dans les préoccupations scientifiques et politiques. Cette évolution s'explique premièrement par le changement dans la nature des conflits armés, qui sont moins souvent interétatiques qu'internes, d'où une augmentation massive du nombre de victimes civiles. On en est donc peu à peu venu à penser qu'il est impossible d'instaurer une stabilité durable et de prévenir des conflits armés sans garantir un minimum de sécurité individuelle. Deuxièmement, il est apparu que la prévention des conflits passe aussi par des mesures à long terme qui ne sont pas du seul ressort de la diplomatie et de l'armée, mais relèvent de plus de l'économie, de la politique et de la situation sociale. Le développement économique et social des individus a aussi gagné en importance dans la politique de sécurité, car il fait partie intégrante d'un ensemble de conditions susceptibles de prévenir les conflits. En parallèle et troisièmement, les acteurs du développement ont pris davantage conscience du rôle de la coopération au développement pour la sécurité. On considère ainsi que garantir des conditions de vie sûres et stables à un individu constitue un prérequis indispensable au développement économique, social et politique d'un pays.

Le dénominateur commun de ces changements de points de vue dans les domaines de la sécurité et du développement, c'est que les gens ont admis qu'il ne peut y avoir de développement sans sécurité, ni de sécurité sans développement. La sécurité humaine (human security) est dès lors au centre de préoccupations communes ${ }^{19}$.

C'est dans le Rapport mondial sur le développement humain 1994 que la notion de sécurité humaine a été largement utilisée pour la première fois ${ }^{20}$. Le rapport énumère sept grands domaines, souvent sujets à risques, dans lesquels la sécurité humaine devrait être garantie: sécurité économique, sécurité alimentaire, sécurité sanitaire, sécurité environnementale, sécurité personnelle, sécurité de la communauté et sécurité politique. Pour simplifier, la notion définie par le PNUD revendique le droit pour chaque individu de vivre à l'abri de la peur et du besoin (freedom from fear, freedom from want). La définition proposée par le PNUD a donné lieu à de nombreuses publications, qui se livrent à une analyse critique de cette nouvelle notion et en donnent de nouvelles définitions. Dans la pratique, on distingue aujourd'hui une interprétation étroite de la sécurité humaine, qui se limite aux aspects liés à la violence (freedom from fear), et une interprétation plus large, qui inclut différents éléments du développement humain. Les deux approches ont en commun de placer l'individu et son besoin de sécurité au centre des préoccupations.

Le Human Security Center de l'University of British Columbia entend élaborer chaque année un Human Security Report pour pouvoir mesurer la sécurité

19 Daniel Trachsler, «Menschliche Sicherheit: Konzept und Praxis », in Andreas Wenger (éd.), Bulletin 2003 zur schweizerischen Sicherheitspolitik, Centre de recherche sur la sécurité et les conflits, Zurich, EPFZ, 2003.

20 Rapport mondial sur le développement humain 1994, PNUD, New York, 1994. 
humaine et son évolution ${ }^{21}$. Ce rapport, qui se concentre sur les aspects liés à la violence dans la sécurité humaine, devrait venir compléter le Rapport mondial sur le développement humain du PNUD.

\section{$\square$ La sécurité humaine dans la politique étrangère de la Suisse}

Aux côtés d'autres pays ${ }^{22}$, la Suisse a aussi commencé à se préoccuper de la sécurité humaine. Cependant, aucune définition commune n'a pour l'heure été adoptée, ce que les commentateurs ne manquent pas de critiquer $^{23}$. Le Rapport sur la politique extérieure 2000 se réfère au Rapport du millénaire du secrétaire général de l'ONU, Kofi Annan, pour présenter la sécurité humaine mondiale: «Ce concept comprend non seulement un monde libéré de la peur (Agenda pour la sécurité), mais également une vie à l'abri du besoin (Agenda pour le développement) ainsi qu'un avenir durable (Agenda pour l'environnement). [...] L'amélioration de la sécurité humaine n'est possible que si les principes de la bonne gestion des affaires publiques sont appliqués. ${ }^{24}$ L'expression revient dans d'autres textes, mais elle n'est jamais clairement définie ni illustrée par des exemples concrets.

Lorsque la Direction du développement et de la coopération utilise cette notion, elle se réfère à son interprétation globale: «La sécurité doit être comprise dans un sens beaucoup plus large: il s'agit du bien-être de tout un chacun. [...] Les fondements de toute politique de sécurité efficace reposent avant tout sur la lutte contre la pauvreté. ${ }^{25}$ On peut dès lors se demander si toute forme de coopération au développement qui vise directement l'individu ne s'inscrit pas dans le domaine de la sécurité humaine.

Toutefois, si c'est la Division politique IV du DFAE qui parle de sécurité humaine, elle se réfère uniquement à l'idée de «vivre à l'abri de la violence» : «A l'instar de la plupart des pays du Nord, la Suisse se préoccupe plus particulièrement des aspects de la sécurité humaine liés à la violence.» Le DFAE a perçu relativement tôt l'utilité de cette notion dans certaines approches. ${ }^{26}$ Parmi les premières activités dans le cadre desquelles la Suisse s'est distinguée en défendant la sécurité humaine, il faut mentionner le combat contre les mines antipersonnel qui a remporté un succès international puisqu'il s'est conclu par la signature de la Convention sur l'interdiction des mines antipersonnel (Convention d'Ottawa de 1997). En 2001, le DFAE a regroupé ses Divisions politiques IV et IIIb pour créer la «Division politique IV, Sécurité humaine (paix, droits de l'homme, politique humanitaire)». Le nom officiel de cette division illustre l'importance que le DFAE accorde à la notion de sécurité humaine, au titre de

21 Human Security Center, University of British Columbia, <www.ligi.ubc.ca/chs.htm>; Human Security Report, <www.humansecurityreport.info $>$. Ce rapport bénéficie notamment du soutien du Réseau de la sécurité humaine et de la Suisse. Au moment de la rédaction du présent Annuaire, sa première édition n'avait pas encore paru.

22 Le Canada et le Japon jouent ici le rôle de chefs de file. Voir aussi <www.humansecurity.gc.ca>.

23 Voir Fred Tanner, «Menschliche Sicherheit - neue Diplomatie», Neue Zürcher Zeitung, 13 janvier 2003; Daniel Trachsler, «Menschliche Sicherheit: Konzept und Praxis», in Andreas Wenger (éd.), op. cit.

24 Rapport du millénaire du secrétaire général de l'ONU, <www.un.org/millennium/sg/report/>; Conseil fédéral, Rapport sur la politique extérieure 2000 - Présence et coopération: la sauvegarde des intérêts dans un monde en cours d'intégration, Berne, 2000.

25 DDC, Thème annuel 2003 "La sécurité par le développement», conférence de presse, 4 février 2003.

26 Peter Maurer, «Die Zivilbevölkerung besser schützen», Neue Zürcher Zeitung, 2 décembre 2002. 
laquelle la Suisse mène aujourd'hui des activités dans différents domaines: lutte contre la prolifération des armes légères, soutien à la Cour pénale internationale, smart sanctions, enfants dans les conflits armés, intégration d'acteurs armés non gouvernementaux, droit international public, rôle des entreprises multinationales dans les conflits armés et respect de codes de conduite ${ }^{27}$. La Suisse compte par ailleurs parmi les membres actifs du Réseau de la sécurité humaine (Human Security Network), au sein duquel elle a lancé l'idée de publier un News Bulletin, et soutient des projets de recherche sur la sécurité humaine ${ }^{28}$.

\section{$\square$ Réseau de la sécurité humaine}

Les ministres des Affaires étrangères du Réseau international de la sécurité humaine se sont retrouvés en mai 2003 en Autriche à l'occasion de la cinquième réunion ministérielle de ce réseau ${ }^{29}$. Les débats ont essentiellement porté sur deux thèmes: l'éducation aux droits de l'homme et les enfants dans les conflits armés. Les ministres ont poursuivi l'élaboration de stratégies politiques pour contribuer à l'éducation aux droits de l'homme et à la sécurité humaine, ainsi que pour protéger les enfants des conséquences de conflits armés et renforcer la présence active du réseau dans certaines conférences internationales ${ }^{30}$. Au sein de ce réseau, la Suisse concentrera à l'avenir ses efforts sur le renforcement du droit humanitaire international et sur le rôle des entreprises multinationales dans les situations de conflit ${ }^{31}$.

\section{$\square$ Armes légères}

A l'occasion de la Conférence internationale sur les armes légères, réunie en juillet 2003 à New York, les participants ont tiré un bilan intermédiaire de deux années d'application du Programme d'action des Nations unies sur les armes légères (non contraignant) ${ }^{32}$. Une centaine de pays ont ainsi rendu compte de leurs programmes nationaux sur les mesures prises pour combattre le trafic d'armes légères et pour éviter les abus.

Chaque année, les armes légères font un demi-million de victimes humaines. Nombre de pays placent dès lors leur contrôle en bonne place dans leurs efforts pour garantir la sécurité humaine. Cette prise de conscience est toutefois contrebalancée par le poids de certains intérêts économiques. La production d'armes légères pèse en effet 7,4 milliards de dollars par an et le lobby des armes est très

27 Voir Conseil fédéral, Message concernant l'ouverture d'un crédit-cadre pour des mesures de gestion civile des conflits et de promotion des droits de l'homme du 23 octobre 2002 (FF 2002 7395).

28 Human Security Network News Bulletin, <www.hsph.harvard.edu/hpcr/human_security.htm>; p. ex. la publication Putting People First: Human Security Perspectives on Small Arms Availability and Misuse, Centre for Humanitarian Dialogue, juillet 2003, <www.hdcentre.org/Programmes/small.htm>.

29 Voici les pays qui sont membres du réseau: Canada, Norvège, Autriche, Slovénie, Grèce, Irlande, Pays-Bas, Chili, Thaillande, Jordanie, Mali, Suisse et Afrique du Sud (en tant qu'observateur). Réseau de la sécurité humaine: <www.humansecuritynetwork.org $>$.

30 Voici les initiatives adoptées lors de la réunion: Déclaration de Graz sur les principes de l'éducation aux droits de l'homme et la sécurité humaine, Manuel sur l'éducation aux droits de l'homme; Combler les lacunes - Stratégie de soutien pour les enfants affectés par les conflits armés; Programme de formation sur les droits de l'enfant pour assurer leur suivi et leur réhabilitation dans les interventions sur le terrain.

31 DFAE, communiqué de presse, 7 mai 2003.

32 Première réunion biennale des Etats sur l'exécution du Programme d'action des Nations unies sur les armes légères, <http://disarmament.un.org:8080/cab/salw-2003.html>. 
influent dans certains pays $^{33}$. Seul l'ajout de règles contraignantes au Programme d'action de l'ONU permettra aux accords sur le contrôle des exportations et sur le marquage des armes légères de porter leurs fruits ${ }^{34}$.

La Suisse et la France ont lancé ensemble un projet de marquage et de traçabilité des armes légères, qui devrait permettre de mieux suivre l'évolution des stocks et les flux d'armement et de jeter les bases de la lutte contre leur trafic. Les deux pays ont réalisé des études de faisabilité et organisé des séminaires sur ce sujet. Un groupe d'experts institué par l'ONU, dont la Suisse fait partie, a conclu en juillet 2003 qu'il est possible et souhaitable d'élaborer un instrument international pour assurer le marquage et la traçabilité des armes légères. Dans ce domaine, la Suisse a par ailleurs financé des études de l'Institut des Nations unies pour la recherche sur le désarmement (UNIDIR) et de l'équipe genevoise de chercheurs «Small Arms Survey» qui a publié l'édition 2003 de l'Annuaire sur les armes légères ${ }^{35}$. Cette édition s'articule autour d'une étude consacrée aux conséquences des armes légères sur le développement humain. Les auteurs parviennent à la conclusion que la disponibilité d'armes légères et les abus dans leur utilisation entravent non seulement le développement humain mais aussi les efforts de développement entrepris par les donateurs, les ONG et les Etats.

L'engagement de la Suisse sur le plan international contraste toutefois singulièrement avec sa politique intérieure ${ }^{36}$. Comparée à d'autres pays, la Suisse présente en effet une forte densité d'armes légères et sa législation en la matière est très permissive. Même après la réforme de l'armée, les citoyens soldats devront conserver leur fusil d'assaut à leur domicile et se verront offrir leur arme d'ordonnance au terme de leur service militaire. La Campagne suisse contre les armes légères, qui plaide en particulier pour la suppression du droit de posséder une arme, a invité le ministre de la Défense, Samuel Schmid, en juillet 2003 à détruire les armes excédentaires de l'armée au lieu de les revendre ${ }^{37}$.

Une révision partielle, actuellement en procédure de consultation, de la loi de 1999 sur les armes devrait combler les lacunes existantes ${ }^{38}$. Elle prévoit notamment de contrôler le commerce des armes à feu, d'interdire leur production en série et d'assurer l'enregistrement de toutes les armes à feu privées dans un registre central.

En octobre 2003, Amnesty International, Oxfam et le Réseau d'action international contre les armes légères (IANSA, International Network on Small Arms) ont lancé dans plus de 60 pays une campagne contre le commerce non contrôlé d'armes à feu ${ }^{39}$. Selon les initiants, bien que la prolifération et les abus dans le domaine des armes soient à l'origine de nombre de décès et de lésions, le commerce international des armes n'est guère contrôlé. La campagne plaide en

\footnotetext{
«One Victim Every Minute», The Economist, 23 juillet 2003.

SPICE-Newsletter, $\mathrm{n}^{\circ}$ 4, 2003, <www.gtz.de/spice>.

5 Annuaire sur les armes légères, <www.smallarmssurvey.org $>$.

36 «Die Schweiz kann in diesem Bereich (internationale Kontrolle von Kleinwaffen) nur dann glaubwürdig argumentieren, wenn sie selber eklatante Lücken in der eigenen Waffengesetzgebung geschlossen hat. » Neue Zürcher Zeitung, 27 septembre 2003.

37 Schweizerischer Friedensrat (Conseil suisse de la paix), Campagne contre les armes légères, <www. friedensrat.ch/kleinwaffen.hauptseite.html>

38 DFJP, Communiqués de presse, 23 septembre 2002, 22 septembre 2003.

39 «Waffen unter Kontrolle», <www.oxfam.de/article.asp?id=361>.
} 
faveur de la conclusion d'un accord international contraignant qui soumette tous les transferts d'équipements d'armement à un contrôle strict et interdise tout commerce d'armes contrevenant au droit international et aux droits de l'homme. Les gouvernements sont également invités à mieux assumer leurs responsabilités au niveau national, à renforcer les contrôles des transferts d'armes et à mieux protéger les citoyens contre les conflits armés. En Suisse, Amnesty International appuie les propositions du Conseil fédéral visant à enregistrer tous les achats et toutes les ventes d'armes à feu ${ }^{40}$.

\section{$\square$ Mines antipersonnel}

La $5^{\text {e }}$ Conférence des Etats parties à la Convention d'Ottawa sur l'interdiction des mines antipersonnel s'est réunie en septembre 2003. Ce fut l'occasion de vérifier l'application de la convention et de débattre d'autres actions ${ }^{41}$. Les Etats représentés à la conférence ont réaffirmé leur volonté de respecter leurs obligations et d'œuvrer pour étendre la convention au monde entier. Les participants ont cependant refusé d'allouer des moyens supplémentaires pour financer des campagnes contre les mines et pour venir en aide aux personnes blessées par des mines. Sur proposition de la délégation suisse, un groupe de travail informel a été chargé de se pencher sur le problème de l'utilisation de mines par des groupes armés non étatiques. Ces derniers ne pouvant pas être soumis aux exigences de la Convention d'Ottawa, l'ONG Appel de Genève négocie depuis 2000 avec des groupes de rebelles pour qu'ils renoncent à utiliser des mines antipersonnel. Jusqu'ici, 22 de ces groupes ont signé la déclaration d'engagement de l'Appel de Genève.

En 2002, la Suisse a consacré plus de 14 millions de francs au combat contre les mines antipersonnel ${ }^{42}$. Près de la moitié de ce montant a été allouée au Centre international de déminage humanitaire - Genève (GICHD). Parmi les pays prioritaires des activités suisses en matière de déminage, on trouve le Mozambique, l'Erythrée, l'Albanie, l'Angola et le Tchad. En 2002, la Confédération a par ailleurs étendu ces activités aux pays suivants: Afghanistan, Angola, Colombie, Ethiopie, Liban et Sri Lanka. En 2002, la plus grande ONG suisse active dans le déminage, la Fondation suisse de déminage, a consacré près de 4 millions de francs à ce travail.

En juin 2003, une querelle a éclaté au sujet de l'organisation d'entraide Menschen gegen Minen $(M g M)$. La fondation allemande $\mathrm{MgM}$ (organisation-mère) a reproché à l'association MgM Suisse de n'avoir investi que 80'000 francs dans des projets de déminage sur les 6,18 millions de francs récoltés entre 2000 et 2002. MgM Suisse n'a pas nié, mais a justifié cet état de fait par les coûts que représente la mise sur pied d'une organisation. Depuis, l'association poursuit ses activités sous le nom d' «Ensemble contre les mines ${ }^{43}$.

40 Amnesty International Section Suisse, communiqué de presse, Lausanne, 9 octobre 2003.

41 Le Landmine Monitor Report 2003 de la Campagne internationale pour interdire les mines passe en revue les progrès et les revers de la lutte pour un monde sans mines antipersonnel, <www.icbl.org/lm $>$.

42 Landmine Monitor Report 2003, <www.icbl.org/lm>.

43 Tagesanzeiger, 28 juin 2003. 
En mars 2003, le Conseil des Etats a été le deuxième conseil à approuver l'ouverture d'un crédit-cadre de 17 millions de francs pour cinq ans destiné à soutenir l'application de la Convention sur les armes chimiques (CAC). La majeure partie de la somme servira à financer des projets d'élimination d'armes chimiques en Russie.

메 Annuaire 2003, $\mathrm{n}^{\circ}$ 1, point 11.7., «Désarmement chimique» (p. 105).

Un forum sur la destruction d'armes chimiques, organisé à Genève en juin 2003 par Green Cross et le DFAE, s'est penché sur le problème de l'élimination de l'arsenal chimique de la Russie. La manifestation avait pour objectif de structurer davantage les efforts de désarmement et de mieux coordonner les activités des partenaires internationaux, dont le nombre ne cesse de croître ${ }^{44}$. Il s'agit surtout d'éviter de commettre des erreurs d'investissements, toujours coûteuses.

A l'occasion de cette conférence, le DFAE a publié son dépliant Armes chimiques - Swiss Update, qui présente la Convention sur les armes chimiques et la contribution de la Suisse à son application ${ }^{45}$.

La Confédération a par ailleurs décidé de participer au «Partenariat mondial contre la prolifération des armes de destruction massive ${ }^{46}$. Fondé par les Etats membres du G8, ce partenariat a pour objectif d'empêcher les terroristes, et ceux qui leur accordent refuge, d'accéder à des armes de destruction massive. Les Etats fondateurs ont déclaré consacrer jusqu'à 20 milliards de dollars pour réaliser cet objectif. Les fonds seront affectés en premier lieu à des projets en Russie (désarmement chimique, démantèlement des anciens sous-marins nucléaires, emplois civils pour les scientifiques qui ont travaillé dans le domaine des armes de destruction massive).

Le partenariat offrira à la Suisse de meilleures possibilités pour coordonner ses activités et un cadre plus propice à ses projets, sans l'obliger à s'acquitter d'obligations financières supplémentaires.

\subsection{Armée XXI: éléments de la politique de paix et de sécurité}

Le 18 mai 2003, le peuple et les cantons ont clairement dit oui aux projets de modification de la loi fédérale sur l'armée et l'administration militaire et de la loi fédérale sur la protection de la population et sur la protection civile. La Confédération a donc reçu le feu vert pour lancer le projet Armée XXI et réformer la protection civile. La nouvelle armée, adaptée à l'évolution de la politique de sécuritée ${ }^{47}$, sera plus petite et moins coûteuse. On accroîtra par ailleurs sa souplesse, sa multifonctionnalité et son interopérabilitét ${ }^{48}$.

DD Annuaire 2002, (pp. 215-216); 2001, (pp. 260-262); 2000, (pp. 224-225).

44 Green Cross Suisse, Magazine, $\mathrm{n}^{\circ}$ 2, 2003; DFAE, Armes chimiques - Swiss Update, juillet 2003.

45 DFAE, communiqué de presse, 25 juin 2003.

46 DFAE, communiqué de presse, 28 mai 2003.

47 Cette réforme se fonde sur le rapport de la Commission Brunner (26 février 1998) et sur le rapport du Conseil fédéral sur la politique de sécurité 2000 (7 juin 1999).

48 DDPS, Dossier votations du 18 mai 2003; Rapport du Conseil fédéral sur la conception de l'Armée XXI (Plan directeur de l'Armée XXI) du 24 octobre 2001 (FF 2002 926). 
La plupart des dangers et menaces possédant un caractère international, l'armée intensifie sa coopération internationale dans les domaines de la formation, de l'acquisition d'armements et d'opérations destinées à promouvoir la paix ${ }^{49}$. Dans ces domaines, la Suisse collabore surtout avec les pays voisins ainsi qu'avec la Suède, la Finlande, la Grande-Bretagne et les Etats-Unis ${ }^{50}$. La plupart de ces Etats étant membres du Traité de l'Atlantique Nord, cette coopération vise également l'OTAN.

En créant Armée XXI, la Confédération souhaite participer plus activement à des opérations internationales de promotion de la paix et à des interventions de l'aide humanitaire ${ }^{51}$. L'utilisation de moyens militaires se limitera aux domaines suivants: protection, logistique, communication et sauvetage. Dans le cadre de l'aide humanitaire, les ressources de l'armée seront attribuées à une organisation civile qui sera chargée de la coopération. Malgré la profonde réforme prévue, la tâche centrale de l'armée consistera toujours à défendre la Suisse contre des menaces militaires.

\section{SOURCES}

Confédération suisse, Swiss Peace Supporter, Journal des contributions suisses à la promotion internationale de la paix, Berne, éditions 2003.

DFAE, Federalism, Politorbis la Revue du Centre d'analyse et de prospective, $\mathrm{n}^{\circ} 32$ (1/2003).

DFAE, Conférence de la Division politique IV, Sécurité humaine - Sri Lanka. Document final, 2003.

Amnesty International et Oxfam, Vies brisées - Plaidoyer pour un contrôle renforcé des ventes d'armes à l'échelon international, 2003, <www.controlarms.org $>$.

Centre pour la promotion de la paix, KOFF Newsletter, Berne, éditions 2003.

Fondation suisse de déminage, Rapport annuel 2002, Genève, 2003.

Human Rights Watch, Landmine Monitor Report 2003 : Toward a Mine-Free World, 2003, <www.icbl. org/lm>.

Peace Brigades International, Rundbrief 01/03, Hamburg, 2003.

Dahinden Martin, «Die Schweiz und die Ächtung der Personenminen», in Wenger Andreas (éd.), Bulletin 2003 zur schweizerischen Sicherheitspolitik, Centre de recherche sur la sécurité et les conflits, Zurich, EPFZ, 2003.

Institut universitaire de hautes études internationales, Annuaire sur les armes légères 2003 - Impasse sur le développement, Genève, 2003, <www.smallarmssurvey.org>

Program on Humanitarian Policy and Conflict Research, Human Security Network News Bulletin, vol. 2, $\mathrm{n}^{\text {os }} 1-3,<$ www.hsph.harvard.edu/hpcr/human_security. htm $>$.

Ribaux, Claude André, Friedensförderung und Konfliktbearbeitung in der Schweiz. Eine Bestandesaufnahme, Saint-Gall, Typotron, 2003.

Trachsler Daniel, «Menschliche Sicherheit: Konzept und Praxis», in Wenger Andreas (éd.), Bulletin 2003 zur schweizerischen Sicherheitspolitik, Centre de recherche sur la sécurité et les conflits, Zurich, EPFZ, 2003.

49 Il est également prévu de renforcer la collaboration entre les services de renseignement et les partenaires en Suisse et à l'étranger. DDPS, communiqué de presse, 26 septembre 2003.

50 Pour ce qui est de la coopération, la Suisse a conclu en septembre 2003 divers accords avec la France et l'Allemagne. DDPS, communiqués de presse, septembre 2003.

51 Dans ce contexte, le Conseil national et le Conseil des Etats ont décidé, à une grande majorité, de prolonger l'engagement de la Swisscoy au Kosovo jusqu'à fin 2005 (affaire 03.024 des Chambres fédérales). 


\section{SITES INTERNET}

Amnesty International Section suisse: <www.amnesty.ch $>$.

Appel de Genève: <www.genevacall.org/home.htm>.

Assemblée fédérale - Le parlement suisse: <www.parlament.ch $>$.

Berghof Forschungszentrum für konstruktive Konfliktbearbeitung : <www.berghof-Centre.org/>.

Brigades de paix internationales: <www.peacebrigades.org $>$.

Campagne internationale pour interdire les mines (ICBL) : <www.icbl.org $>$.

Campagne internationale pour le contrôle des armes: $<w w w . c o n t r o l a r m s . o r g>$.

Campagne suisse contre les mines antipersonnel: <www.uxo.ch $>$.

Centre for Humanitarian Dialogue: $<w w w . h d c e n t r e . o r g>$.

E-Mine, The Electronic Mine Information Network: $<w w w . m i n e a c t i o n . o r g>$.

Fondation suisse pour la paix, Swisspeace: $<w w w . s w i s s p e a c e . o r g\rangle$.

Green Cross Suisse: <www.greencross.ch>.

International Action Network on Small Arms : <www.iansa.org >.

Oxfam: <www.oxfam.org>.

Réseau de la sécurité humaine: <www.humansecuritynetwork.org>. 